\title{
Oral Health Related Quality of Life in Adult Population Attending Outpatient Department of KD Dental College and Hospital, Mathura
}

\author{
Sirohi $\mathbf{R}^{1}$, Kaur $\mathbf{N}^{2}$, Ingle NA ${ }^{3}$, Siwach $\mathbf{A}^{4}$
}

\section{ABSTRACT}

The concept of oral health related quality of life (OHRQOL) attempts to characterize patients's perception of oral health. Currently there is a growing interest in oral health outcomes in how oral health affects quality of life.

\section{OBJECTIVE}

To assess oral health related quality of life in adult population attending the Outpatient Department of K.D Dental College and Hospital, Mathura.

\section{MATERIALS AND METHOD}

A cross sectio nal study was carried out for a period of one month in OPD of K.D Dental College and Hospital, Mathura. Three twenty individuals were selected randomly. An investigation was done by using a combination of questionnaire and clinical examination of dental caries was done by using WHO 1997 criteria.

\section{RESULTS}

Majority of the participants (70.9\%) reported oral health problems affecting at least one daily performance in six months preceding the survey. The performance most affected was on 'eating' (52.8\%) followed by 'cleaning teeth' (29.7\%). Chronbach's alpha for the OIDP frequency items was 0.70.

\section{CONCLUSION}

The highest impact was 'eating' and lowest on 'speaking and pronouncing' in the study population, which in turn, had an impact on their Quality of Life.

KEYWORDS: Oral health, Oral health related quality of life, Oral impact on daily performance.

${ }^{1}$ Senior Lecturer
Department of Public Health Dentistry
Kalka Dental College, Meerut, UP, INDIA
${ }^{2}$ Reader
Department of Public Health Dentistry
KD Dental College, Mathura, UP, INDIA

KD Dental College, Mathura, UP, INDIA

\author{
${ }^{3}$ Professor and Head \\ Department of Public Health Dentistry \\ KD Dental College, Mathura, UP, INDIA \\ ${ }^{4}$ Senior Lecturer \\ Department of Prosthodontics \\ Kalka Dental College, Meerut, UP, INDIA
}

\section{Contact Autbor}

Dr. Reenu Sirohi

dr.reenu.08@gmail.com

\section{INTRODUCTION}

ral health is an integral part of general health and essential for well-being of an individual. Quality of life is a broad concept concerned with the overall well-being in the society. The World Health Organization defines Quality of life as an "individual's perception of their position in life in the context of the culture and value systems in which they live and relation to their goals, exceptations, standards and concerns" 
(1). The use of health related quality of life refers to the extent to which one's usual or expected physical, emotional and social well-being are affected by a medical condition or its treatment (2).

Health is no longer defined in terms of illness and disease but the concept has been broadened to take into account physical, physiological and social aspect of well-being (WHO 1946). It is now well recognized that there are serious limitations in solely using the clinical normative assessments for the measurement of oral health status and need, as they do not consider the individuals perceived health status or needs. Hence, a variety of socio-dental indicators or oral health related quality of life measures (OHRQoL) have been advocated in the past 20 years which capture people's perceptions about factors that are important in their everyday lives and complement clinical conventional assessments. They include the Geriatric (General) Oral Health Assessment Index (GOHAI), Child Oral Health Quality of Life Questionnaires (COHQOL), Oral Health Impact Profile (OHIP) and Oral Impacts on Daily Performance (OIDP) (3).

The Oral Health Impact Profileshort form (OHIP-14) is one of the OHRQoL instruments that has been widely used in several cross-sectional and longitudinal studies. When considering the methods of administration, the use of questionnaires in research to assess OHRQoL presents substantial advantages such as lower cost, preservation of the anonymity of the participants and reduction of biases that can occur in the interaction with the interviewer. On the other hand, the potential for lower response rates exists when this form of administration is used. The form of administration generally does not appear to influence the total scores of the instrument when it is applied in an elderly population (4).

The OHIP-14, like the original ver- sion, includes seven dimensions. Each dimension contains two questions. The shortened version has a reliability and validity similar to that of the original version and is an appropriate tool for statistical analysis related to the effect of oral health on an individual's quality of life (5).

In Mathura, oral diseases are widely prevalent, often very painful and expensive to treat, causing loss of several working days. Besides poor oral health affects masticatory function, speech and ultimately over all well-being of an individual. However, there is a need to check the oral health related quality of life among adults in Mathura. Therefore, a survey was planned to describe the oral health related quality of life, in adult population attending the outpatient department of K.D Dental College and Hospital, Mathura.

\section{MATERIALS AND METHOD}

A cross sectional study was conducted, which included questionnaire and clinical examination on the study population consisting of three hundred and twenty adults who were conveniently selected from dental patients attending out patient department in K.D Dental college and hospital, Mathura. Prior to start of the study the ethical clearance was obtained from ethical committee of K.D Dental College \& Hospital, Mathura. Before the data collection the purpose and procedure of the study was thoroughly explained to the subjects and written informed consent of each subject was obtained.

Inclusion criterias:

- Those who agreed to participate in the study.

- Those who were in age group of 35-44 years.

- Those who were free from systemic medical conditions, neurological diseases and mental disabilities.

- Those who were not edentulous

Oral Impact on Daily Performance was pilot tested among 30 subjects. According to estimated prevalence of oral impact in pilot study $(83 \%)$, the minimum sample size of 318 subjects was calculated. The OHIP-14 (oral health impact profile) included questions about problems patients might have encountered with their teeth, mouth and denture. The questionnaire had three parts: information on age and gender of participants along with oral health behaviors like frequency of brushing and frequency of dental visits; the OIDP inventory, which included eight physical, social and psychological aspects of daily performance; and perceptions of respondents and satisfaction with dental appearance. The OIDP inventory section asked how often during the past 6 months patients mouth and teeth caused any difficulty with: 'eating and enjoying food', 'speaking and pronouncing clearly', 'cleaning teeth', 'sleeping and relaxing', 'smiling and showing teeth without embarassement', 'maintaining usual emotional state', 'carrying out major work and social role' and 'enjoying contact with people'. Regarding percieved general health,oral health and satisfaction with dental appearance a three point response scale was coded as (1) Satisfied (2) Average (3) Dissatisfied.

The clinical examination of all patients involved in the study was done by single examiner, the invigilator. Subjects were examined under adequate illumination ( Type III) and clinical data were collected on dental caries, missing teeth and filled teeth using the DMFT index (WHO 1997) (6). Dental probes and plane mouth mirrors were used to assess carious lesions.

A five - point Likert scale was used to measure the responses to OHIP-14 (oral health impact profile) items with the possible score ranging from 0-56. The reliability of the instrument was assessed by standardized chronbach's alpha, inter item and item total correlation coefficients. As the OIDP scores were not normally distributed, testing for criterion and construct validity was 
carried out using non-parametric tests; Mann-Whitney and Kruskal-Wallis as applicable. All statistical analysis were carried out using the statistical package for social sciences, version 15 software package.

\section{RESULTS}

Out of three hundred and twenty study participants who agreed to participate in the study, two hundred and twenty four i.e $70 \%$ were males and ninety six i.e $30 \%$ were females. Among three hundred and twenty participants who responded, one hundred and sixty six i.e $52.8 \%$ were affecting OIDP inventory as not satisfactory according to "difficulty in eating" aspect. It was the most prevalent impact, reported by approximately half of the respondents. (Table.1). The OIDP instrument showed very good reliability and demonstrated homogenecity of items with chronbach's alpha of 0.70. All corrected item-total correlations were above the minimum recommended level of 0.02 to be included in the scale. All were positive and no correlation was high enough for any item to be redundant. (Table.2) Construct validity of the instrument demonstrated that the mean OIDP score showed a clear trend with OIDP scores; those with perceived 'dissatisfaction with general health', 'oral health', 'dental appearance' having higher OIDP score, indicating high level of oral impacts $(p<0.001)$. Criterion validity of the instrument demonstrated that mean OIDP scores were significantly associated with decayed teeth and missing teeth but insignificantly associated with filled teeth and DMFT scores. (Table.3)

\section{DISCUSSION}

Out of three hundred and twenty study participants who were willing to participate in the study, $70 \%$ were males and $30 \%$ females. On the contrary, in the study conducted by Navin Anand Ingle et al (1) out of the total 307 study subjects, $49.9 \%$ were males and $52.1 \%$ were females. Among three hundred and twenty participants who responded, $52.8 \%$ were affecting OIDP inventory as not satisfactory according to "difficulty in eating" aspect. Similarly, in the study conducted by B.M Purohit et al (3) $52.2 \%$ participants were affecting OIDP inventory as not satisfying according to "difficulty in eating" aspect. It was the most preva-

\begin{tabular}{|c|c|c|c|c|c|c|}
\hline Component of the OIDP inventory & $\begin{array}{c}\text { Every } \\
\text { day }\end{array}$ & $\begin{array}{l}3-4 \text { times } \\
\text { per week }\end{array}$ & $\begin{array}{l}\text { 1-2 times } \\
\text { per week }\end{array}$ & $\begin{array}{c}\text { Once } \\
\text { per month }\end{array}$ & $\begin{array}{c}\text { Total } \\
\text { affected }\end{array}$ & $\begin{array}{c}\text { Never } \\
\text { affected }\end{array}$ \\
\hline Difficulty in eating & 25.3 & 6.8 & 8.9 & 11.8 & $52.8 \%$ & $47.2 \%$ \\
\hline Difficulty in cleaning & 14.1 & 7.0 & 5.2 & 3.4 & $29.7 \%$ & $70.3 \%$ \\
\hline Difficulty in speaking and pronouncing clearly & 3.7 & 4.1 & 1.2 & 0.8 & $9.8 \%$ & $90.2 \%$ \\
\hline Difficulty in sleeping & 4.7 & 4.2 & 2.9 & 8.2 & $20 \%$ & $80 \%$ \\
\hline Difficulty in carrying out major work & 7.8 & 4.1 & 1.9 & 6.2 & $20 \%$ & $80 \%$ \\
\hline Difficulty in smiling and showing teeth without embarrassment & 9.8 & 0.9 & 2.4 & 6.0 & $19.1 \%$ & $80.9 \%$ \\
\hline Difficulty in enjoying contact with people & 9.4 & 1.3 & 1.9 & 6.4 & $19 \%$ & $81 \%$ \\
\hline Difficulty in maintaining usual emotional state & 11.2 & 4.3 & 2.9 & 7.4 & $26.8 \%$ & $73.2 \%$ \\
\hline
\end{tabular}

\section{Table 2: Reliability analysis using chronbach's alpha}

\begin{tabular}{l|c|c} 
OIDP Items & $\begin{array}{c}\text { Corrected item total } \\
\text { correlation }\end{array}$ & $\begin{array}{c}\text { Chronbach's Alpha } \\
\text { if item detected }\end{array}$ \\
\hline Difficulty in eating & 0.39 & 0.57 \\
\hline Difficulty in cleaning & 0.27 & 0.59 \\
\hline Difficulty in speaking and pronouncing clearly & 0.32 & 0.67 \\
\hline Difficulty in sleeping & 0.52 & 0.61 \\
\hline Difficulty in carrying out major work & 0.48 & 0.67 \\
\hline Difficulty in smiling and showing teeth without embarrassment & 0.51 & 0.62 \\
\hline Difficulty in enjoying contact with people & 0.18 & 0.72 \\
\hline Difficulty in maintaining usual emotional state & 0.42 & 0.68 \\
\hline Standardized Chronbach's Alpha & 0.70 & \\
\hline
\end{tabular}


Tabe 3: Construct and criterion validity for OIDP inventory: comparison of mean OIDP with perceived general and oral health, appearance of teeth and caries experience

\begin{tabular}{|c|c|c|c|}
\hline Variable & & OIDP mean (SD) & $\begin{array}{c}\text { p value } \\
\text { (Kruskal-Wallis) }\end{array}$ \\
\hline \multicolumn{4}{|l|}{ Construct validity } \\
\hline \multirow[t]{3}{*}{ Perceived general health status } & Satisfied & $12.4(4.0)$ & \\
\hline & Average & $16.2(6.4)$ & $<0.001$ \\
\hline & Dissatisfied & $19.1(17.9)$ & \\
\hline \multirow[t]{3}{*}{ Perceived oral health status } & Satisfied & $11.2(5.1)$ & \\
\hline & Average & $13.6(5.8)$ & $<0.001$ \\
\hline & Dissatisfied & $18.9(7.2)$ & \\
\hline \multirow[t]{3}{*}{ Perceived satisfaction with appearance of teeth } & Satisfied & $11.7(2.9)$ & \\
\hline & Average & $11.9(5.6)$ & $<0.001$ \\
\hline & Dissatisfied & $17.9(7.8)$ & \\
\hline \multicolumn{4}{|l|}{ Criterion validity } \\
\hline \multirow[t]{2}{*}{ Decayed teeth } & 0 & $11.3(4.9)$ & \\
\hline & $1-5$ & $12.9(6.8)$ & $0.3^{*}$ \\
\hline \multirow[t]{3}{*}{ Missing teeth } & $\geq 6$ & $14.2(6.2)$ & \\
\hline & 0 & $12.3(5.4)$ & \\
\hline & $1-5$ & $13.8(6.4)$ & $0.4^{*}$ \\
\hline \multirow[t]{3}{*}{ Filled teeth } & $\geq 6$ & $14.9(4.0)$ & \\
\hline & 0 & $13.3(4.9)$ & \\
\hline & $1-5$ & $12.9(6.7)$ & 0.67 \\
\hline \multirow[t]{4}{*}{ DMFT } & $\geq 6$ & $12.2(6.1)$ & \\
\hline & 0 & $9.9(2.4)$ & \\
\hline & $1-5$ & $11.9(5.9)$ & 0.07 \\
\hline & $\geq 6$ & $14.1(6.4)$ & \\
\hline
\end{tabular}

lent impact, reported by approximately half of the respondents in both studies. Out of total study participants who responded, $29.7 \%$ were affected OIDP inventory as not satisfactory according to "difficulty in cleaning" aspect. On the contrary, in the studies conducted by Astrom AN et al (7) and B.M Purohit et al (3) impact perceived was 5.4\% and $22.1 \%$ respectively. About $9.8 \%$ of study participants were affected OIDP inventory as not satisfactory for "difficulty in speaking and pronouncing clearly". On the contrary, the studies conducted by Astrom AN et al (7) and B.M Purohit et al (3) impact perceived were only $3.0 \%$ and $14.4 \%$ respectively. About 20\% were affected OIDP inventory as not satisfactory according to "difficulty in sleeping" and "carrying out major work". On the other hand studies done by Astrom AN et al (7) and B.M Purohit et al (3) impact perceived were $3.9 \%$ and $19.2 \%$ respectively for "difficulty in sleeping" aspect and $3.0 \%$ and $16 \%$ for "carrying out major work" aspect of OIDP inventory. In total $19.1 \%$ were affecting OIDP inventory as not satisfactory according to difficulty in smiling and showing teeth without embarrassment, represented social aspect of OIDP inventory. On the contrary, in the studies conducted by Javier Montero- Martin et al (8), $8.7 \%$ participants were affecting OIDP inventory as not satisfying according to "difficulty in smiling and showing teeth without embarrassment" aspect. About 19\% among total three hundred and twenty study participants were affected OIDP inventory as not satisfactory for "difficulty in enjoying contact with people". On the other hand in studies done by Astrom AN et al (7) and B.M Purohit et al (3) impact perceived were 2.1 and $16 \%$ respectively for "difficulty in sleeping" aspect and $3.0 \%$ and $16 \%$ for "carrying out major work" aspect of OIDP inventory. Nearly $26.8 \%$ were affected OIDP inventory as not satisfactory according to "difficulty in maintaining usual emotional states" represented social aspect of daily performance. On the contrary, in the studies conducted by 
Astrom AN et al (7) and B.M Purohit et al (3) impact perceived were $2.1 \%$ and $20.8 \%$ respectively for psychological aspect of OHIPD.

In the present study, it was concluded that OHIP-14 is a precise, valid and reliable instrument for assessing oral health related quality of life among adult population which were in the accordance to the studies conducted by Szentpetery A (9), Nadia Khalifa (10), Mina Molallebnijade (11) and Nikolina Kenig (12). On the other hand, Paula Cristina (13) found that the use of the OHIP-14 in the questionnaire format may result in lower completion rates and loss of data.

The results of the study showed that OHIP-14 scores were significantly associated with oral health status indicators and had an important effect on oral health related quality of life. The highest impact was 'eating' and lowest on 'speaking and pronouncing' in the study population, which in turn, had an impact on their Quality of Life. This study demonstrated a high prevalence of oral diseases in the study population, which in turn, had an impact on their quality of life.

\section{REFERENCES}

1. FH Hirata, $\mathrm{S}$ Kurup, $\mathrm{P}$ John, J, Joley. Oral health and quality of life assessment within Indian population. Amrita J Med 2011;7:17-23.

2. Gella DF, Bonomi AE. Quality of life demands and reality as a medical parameter versus quality of life as individual's reality. Measuring $\mathrm{OHQOL}$ 1995;9:47-60.

3. BM Purohit, A. Singh, S. Acharaya, $M$ Bhat, $H$ Priya. Assessment and validation of the oral impacts on daily performances (OIDP) instrument among adult in Karnataka. Comm Dent Health 2012;29:203-08.

4. Paula Cristina Brolezi de Sousa, Fausto Medeiros Mendes, Jose Carlos Pettorossi Imparato and Thiago Machado Ardenghi. Diffrences in responses to the oral health impact profile (OHIP-14) used as a questionnaire or in an interview. Brazil Oral Res 2009;29:358-64.

5. Slade GD. Derivation and validation of a short-form oral health impact profile. Comm Dent and Oral Epidemiol 1997;25:284-90.

6. World Health Organization: Oral Health Surveys: Basic methods, 4th edition Geneva: WHO 1997.

7. AN Astrom, O Haugejorden, E Skaret, A Trovik, KS Klock. Oral impacts on daily performance in Norwegian adults: the influence of age, number of missing teeth and sociodemographic factors. Europ $J$ Oral Sci 2006;114:115-121.

8. Javier Montero-Martin, Manuel BravoPerez, Alberto Albaladejo-Martin, Eva Maria Rosel-Gallardo. Validation the oral health impact profile (OHIP-14) for adults in Spain. J Clinical Exper Dent 2009;1:1-7.

9. Andras Sczenpetery, Gyula Szabo, Gyula, Marda, Ildiko Szantoand Mike T.John. The Hungarian version of the oral health impact profile. Europ $J$ Oral Sci 2006;114:197-203.

10. Nadia Khalifa Patrick F. Allen, Neamat H. Abu-bakr, Manar E. Abdel-Rahman. Psychometric properties and performance of the oral health impact profile (OHIP. $14 \mathrm{~s}-\mathrm{ar}$ ) among Sudanese adults. J Oral Sci 2013;55:122-32.

11. Mina Motallebnejad, Hoora Hardian, Shervin Mehdizadeh and Mahmoud. Validity and reliability of Persian version of oral health impact profile (OHIP-14). Caspian. J Int Med 2011;2:314-20.

12. Nikolina Kenig, Julijana. Assessing the Psychometric characteristics of the Macedonian version of the oral health impact profile questionnaire (OHIP-MAC 49). OHDM 2012;11:29-38.

13. Paula Cristina Brolezi de Sousa, Fausto Medeiros Mendes, Jose Carlos Pettorossi Imparato and Thiago Machado Ardenghi. Diffrences in responses to the oral health impact profile (OHIP-14) used as a questionnaire or in an interview. Brazil Oral Res 2009;29:358-64. 